\title{
Temporäre räumliche Nähe - Akteure, Orte und Interaktionen
}

https://doi.org/10.2478/rara-2020-0004

In wissensbasierten Ökonomien beruht die Wettbewerbsfähigkeit von Unternehmen insbesondere auf ihrer Fähigkeit, neues Wissen zu erzeugen und dieses mit ihren Partnern auszutauschen. Während sich die Raumwissenschaften mit den räumlichen Besonderheiten des Austausches von Wissen auseinandergesetzt und in diesem Zusammenhang lange Zeit permanente Konzentrationen von Akteuren (z. B. regionale Cluster) analysiert haben, hebt eine wachsende Zahl jüngerer Arbeiten hervor, dass der Austausch von Wissen nicht zwangsläufig räumliche Akteursnähe voraussetzt. Speziell die Arbeiten zu temporären Clustern (Maskell/Bathelt/Malmberg 2004; Henn/Bathelt 2015) und zu „Field Configuring Events" (Lange/Power/Suwala 2014; Schüßler/Grabher/ Müller-Seitz 2015) legen nahe, dass Akteure aus weit voneinander entfernten Standorten oftmals nur für kurze Zeit, zumeist in Städten, zusammenkommen und für Innovationen hochrelevantes Wissen im Rahmen von ,nur' temporär hergestellten Face-to-face-Kontakten austauschen. Als Beispiele für derartige Settings lassen sich Tagungen und Konferenzen (Garud 2008), Ausstellungen und internationale Leitmessen (Bathelt/Schuldt 2010) identifizieren. Doch auch Werkstätten als Orte der Co-Creation (Fleischmann/Hielscher/Merritt 2016; Kohtamäki/Partanen 2016) oder der Zusammenarbeit in Projekten (Torre 2011; Grabher/Thiel 2015) kann in diesem Zusammenhang eine bedeutende Rolle erwachsen.

Während die Forschung zu temporären Formen des Wissensaustausches in den vergangenen Jahren deutlich zugenommen hat (Bathelt/Henn 2014; Schmidt/ Brinks/Brinkhoff 2014; Growe/Mager 2018; Henn/Bathelt 2018; Growe 2019a; Growe 2019b), ist nach wie vor noch nicht hinreichend bekannt, unter welchen Bedingungen Akteure von temporärer räumlicher Nähe profitieren, welche Orte sich zur Durchführung temporärer Settings ganz grundsätzlich anbieten bzw. systematisch genutzt werden und wie derartige Orte bewusst geschaffen und/ oder gestaltet werden können, um den Wissensaustausch zu katalysieren.

Anliegen dieses Schwerpunktheftes der Zeitschrift „Raumforschung und Raumordnung | Spatial Research and Planning" ist es daher, diese Aspekte näher zu betrachten und ausgewählte temporäre Akteurkonstellationen und ihre Bedeutung für betriebliche bzw. regionale Entwicklungsprozesse zur Diskussion zu stellen. Im Zentrum des Erkenntnisinteresses stehen dabei die den Wissensaustausch ermöglichenden Akteure, Orte und Interaktionen.

- In Bezug auf die am Wissensaustausch beteiligten Akteure wird der Frage nachgegangen, mit welchen Beweggründen diese temporäre räumliche Nähe herstellen bzw. wie sie mit Chancen und Herausforderungen, die sich aus temporärer räumlicher Kopräsenz ergeben, umgehen. Damit in Verbindung steht auch die Auseinandersetzung mit der Frage, wie genau Akteure zum Wissenstransfer über geographische Distanzen beitragen.

- Im Zusammenhang mit Orten wird thematisiert, wo genau spontane oder auch bewusst organisierte Treffen zum Austausch ökonomisch relevanten Wissens stattfinden und nach welchen Kriterien diese Orte ausgewählt werden.

- Hinsichtlich Interaktionen wiederum stehen Fragen nach den Wechselwirkungen zwischen physischer Ko-Präsenz und der Nutzung von Informations- und Kommunikationstechnologien bzw. Möglichkeiten zur Intensivierung des Austausches zwischen den betrachteten Akteuren im Fokus.

\footnotetext{
*Corresponding author: Prof. Dr. Anna Growe, Universität Heidelberg, Institut für Geographie, Berliner Straße 48, 69120 Heidelberg, Deutschland, E-Mail: anna.growe@uni-heidelberg.de

Prof. Dr. Sebastian Henn, Friedrich-Schiller-Universität Jena, Institut für Geographie, Löbdergraben 32, 07743 Jena, Deutschland
} 
Konkret widmen sich die folgenden sechs Beiträge den oben genannten Aspekten, wobei von den Autorinnen und Autoren durchaus unterschiedliche Akzentuierungen gewählt wurden:

Anna Butzin und Kerstin Meyer heben die Unterschiede zwischen temporärer räumlicher Nähe in Produktionsprozessen einerseits und in Wissensaustauschprozessen andererseits hervor. Konkret widmen sie sich vier unterschiedlichen Orten produktionsbedingter temporärer räumlicher Nähe: Großunternehmen, Manufakturen, Makerspaces und Festivals. Die Ergebnisse der Untersuchung zeigen, dass produktionsbedingte temporäre räumliche Nähe zwischen Produzentinnen/ Produzenten und Kundinnen/Kunden zwar individuelle Geschäftsmodelle und die Standortwahlprozesse beeinflussen mag, die mit inr in Beziehung stehende innerstädtische Produktion aber nicht imstande ist, die massenhafte Herstellung von Konsumgütern zu ersetzen.

Alice Melchior, Benjamin Schiemer und Gernot Grabher untersuchen in ihrem Beitrag die raumzeitliche Beziehung zwischen auf Kolokation beruhender persönlicher Interaktion und den auf dem Einsatz von Kommunikationstechnologien basierenden Interaktionen. Konkret stehen Arbeitsprozesse in einem pharmazeutischen Labor und in einem Tonstudio im Zentrum ihrer Analyse. Im Ergebnis schlagen die Autoren eine Erweiterung des zeitgeographischen Ansatzes von Torsten Hägerstrand um eine virtuelle Dimension mit der Absicht vor, raumzeitliche Beschränkungen kollaborativer Prozesse empirisch vergleichbar und erfassbar zu machen.

Im Mittelpunkt des Beitrags von Andreas Kuebart und Oliver lbert steht die Auseinandersetzung mit acht unterschiedlichen Formen von Kopräsenz, die sich aufgrund von unterschiedlichen Interaktionsprozessen der beteiligten Akteure im Zusammenhang mit Seed-AcceleratorProgrammen in unterschiedlichen Städten (Amsterdam, Berlin, Detroit und Hamburg) differenzieren lassen. Ihre Analyse belegt, dass Kopräsenz dazu beitragen kann, relationale Distanz zum Nutzen von Gründungsprozessen zu überbrücken.

Der Beitrag von Madeleine Wagner und Anna Growe beschäftigt sich mit der Förderung von Kreativität durch die Schaffung temporärer räumlicher Nähe im SAP AppHaus in Heidelberg. Dabei rücken die innenarchitektonische Gestaltung und Ausstattung des Ortes, an dem temporäre räumliche Nähe realisiert wird (im SAP AppHaus), die Interaktionen auf Workshops zur Realisierung temporärer räumlicher Nähe sowie die Ausrichter und Nachfrager von Workshops im SAPApp-Haus in den Vordergrund der Analysen. Die Autorinnen arbeiten heraus, dass die zeitliche Befristung von Interaktionen auf Work- shops, die neuen Akteurkonstellationen während der Workshops und die Durchführung der Workshops in speziell designten Räumlichkeiten zu einer Eventisierung von Wissensaustausch und Wissensgenerierung führen, die den kreativen Prozess fördert.

Der Beitrag von Nils Pfeufer und Lech Suwala befasst sich mit Fragen der Temporalität und Eventisierung eines spezifischen Ortstypus, den sogenannten Pop-up-Restaurants. Im Zusammenhang mit der Analyse von Standortstrategien dieser Unternehmen veranschaulichen die Autoren, wie sich temporäre Raumnutzungen durch gastronomische Betriebe in Wert setzen lassen. Ihre Analyse belegt, dass dem Betrieb dieser temporären Gastronomien diverse Ziele und Hintergründe zugrunde liegen und sich die Standortstrategien der Unternehmen in unterschiedlichen Formen der Standortwahl artikulieren.

Der abschließende Beitrag von Lena Greinke und Nicola Hilti lenkt den Blick auf die Verbindung von Arbeit und Wohnen in einer zunehmend wissensbasierten Gesellschaft. Es werden temporär genutzte Räume von berufsbedingt multilokal lebenden Personen analysiert. Der Beitrag erörtert insofern nicht die Bedeutung temporärer räumlicher Nähe in Wirtschaftsprozessen, sondern deren Relevanz für Lebenssituationen, die durch eine zunehmende Flexibilisierung im Schnittbereich von Arbeits- und Privatleben gekennzeichnet sind. Auf dieser Grundlage werden Herausforderungen und Chancen für ländliche Räume diskutiert. Im Einzelnen verdeutlichen die Autorinnen, dass sich das Altags- und Berufsleben in den temporär genutzten Räumen sehr divers gestaltet und multilokale Lebensweisen von betrieblichen Rahmenbedingungen und Strukturen beeinflusst werden.

Alles in allem setzen sich die Beiträge dieses Schwerpunktheftes mit temporärer Nähe in völlig unterschiedlichen räumlichen Konstellationen auseinander, die von Tonstudios bis hin zu Pop-Up-Restaurants reichen. Mit dem Fokus auf temporär genutzten Räumen von berufsbedingt multilokal Lebenden wird zudem der Blick über Wirtschaftsakteure hinaus erweitert und das Spannungsfeld von Flexibilität zwischen Berufs- und Privatleben einbezogen. Als Koordinatoren dieses Schwerpunktheftes ist uns bewusst, dass hier die oben andiskutierte Rolle von Akteuren, Orten und Interaktionen im Zusammenhang mit Fragen temporärer Akteurnähe keinesfalls abschließend behandelt wird. Gleichwohl sind wir auch davon überzeugt, dass die Beiträge - jeweils für sich genommen oder in ihrer spezifischen Zusammenstellung - einen wichtigen Anstoß für weiterführende Diskussionen und Analysen geben können. 


\section{Literatur}

Bathelt, H.; Henn, S. (2014): The geographies of knowledge transfers over distance: toward a typology. In: Environment and Planning A 46, 6, 1403-1424. doi: 10.1068/a46115

Bathelt, H.; Schuldt, N. (2010): International Trade Fairs and Global Buzz, Part I: Ecology of Global Buzz. In: European Planning Studies 18, 12, 1957-1974. doi: 10.1080/09654313.2010.515815

Fleischmann, K.; Hielscher, S.; Merritt, T. (2016): Making things in Fab Labs: a case study on sustainability and co-creation. In: Digital Creativity 27, 2, 113-131. doi: 10.1080/14626268.2015.1135809

Garud, R. (2008): Conferences as Venues for the Configuration of Emerging Organizational Fields: The Case of Cochlear Implants. In: Journal of Management Studies 45, 6, 1061-1088. doi: 10.1111/j.1467-6486.2008.00783.x

Grabher, G.; Thiel, J. (2015): Projects, people, professions: Trajectories of learning through a mega-event (the London 2012 case). In: Geoforum 65, 328-337. doi: 10.1016/j. geoforum.2015.02.006

Growe, A. (2019a): Buzz at workplaces in knowledge-intensive service production: Spatial settings of temporary spatial proximity. In: European Urban and Regional Studies 26, 4, 434-448. doi: 10.1177/0969776418784999

Growe, A. (2019b): Developing trust in face-to-face interaction of knowledge-intensive business services (KIBS). In: Regional Studies 53, 5, 720-730. doi: 10.1080/00343404.2018.1473567

Growe, A.; Mager, C. (2018): Changing workplaces in the creative process in creative industries - the case of advertising and music. In: Erdkunde 72, 4, 259-271. doi: 10.3112/ erdkunde.2018.04.01

Henn, S.; Bathelt, H. (2015): Knowledge generation and field reproduction in temporary clusters and the role of business conferences. In: Geoforum 58, 104-113. doi: 10.1016/j. geoforum.2014.10.015

Henn, S.; Bathelt, H. (2018): Cross-local knowledge fertilization, cluster emergence, and the generation of buzz. In: Industrial and Corporate Change 27, 3, 449-466. doi: 10.1093/icc/dtx036

Kohtamäki, M.; Partanen, J. (2016): Co-creating value from knowledge-intensive business services in manufacturing firms. The moderating role of relationship learning in suppliercustomer interactions. In: Journal of Business Research 69, 7, 2498-2506. doi: 10.1016/j.jbusres.2016.02.019

Lange, B.; Power, D.; Suwala, L. (2014): Geographies of fieldconfiguring events. In: Zeitschrift für Wirtschaftsgeographie 58, 1, 187-201. doi: 10.1515/zfw.2014.0013

Maskell, P.; Bathelt, H.; Malmberg, A. (2004): Temporary Clusters and Knowledge Creation: The Effects of International Trade Fairs, Conventions and Other Professional Gatherings. Marburg.

Schmidt, S.; Brinks, V.; Brinkhoff, S. (2014): Innovation and creativity labs in Berlin: Organizing temporary spatial configurations for innovations. In: Zeitschrift für Wirtschaftsgeographie 58, 4, 232-247. doi: 10.1515/zfw.2014.0016

Schüßler, E.; Grabher, G.; Müller-Seitz, G. (2015): Field-Configuring Events. Arenas for Innovation and Learning? In: Industry and Innovation 22, 3, 165-172. doi: 10.1080/13662716.2015.1038098

Torre, A. (2011): The role of proximity during long-distance collaborative projects. Temporary geographical proximity helps. In: International Journal of Foresight and Innovation Policy 7, 1/2/3, 213-230. doi: 10.1504/IJFIP.2011.040075 